\title{
TÉCNICA PROCESAL PARA EL CUMPLIMIENTO PROVISIONAL DE DECISIONES JUDICIALES EN EL DERECHO PROCESAL CIVIL BRASILEÑO
}

\author{
Procedural technique for provisional enforcement of the judicial \\ decisions in Brazilian civil procedural law
}

\begin{abstract}
Daniel Roberto Hertel
Profesor Adjunto de Derecho Procesal Civil y Práctica Jurídica Civil en

la Universidad de Vila Velha

Profesor visitante de Derecho Procesal Civil en el Programa de Posgrado de la Academia Brasileña de Derecho Constitucional Profesor de la Escuela de la Magistratura del Estado de Espírito Santo danielhertel@terra.com.br; daniel@uvv.br
\end{abstract}

Recibido: 21/07/2020 - Aceptado: 03/08/2020

\begin{abstract}
Resumen
Trata de los diversos aspectos relacionados con el cumplimiento provisional (ejecución provisional) de las decisiones judiciales en el Código de Proceso Civil Brasileño de 2015 (CPC), centrándose en los precedentes del Superior Tribunal de Justicia (STJ) y en los precedentes del Supremo

- Palabras clave: Cumplimiento provisional; Decisión judicial; Procedimiento

Tribunal Federal (STF). Aborda el propósito del cumplimiento provisional, y luego analiza las hipótesis de la idoneidad de esta modalidad de ejecución, contrastándolas con situaciones de cumplimiento definitivo. Se ocupa de las normas de tramitación del cumplimiento provisional, analizando la necesidad de proporcionar seguridad, aclarando sobre el momento procesal exacto para la práctica de ese acto. Las posibilidades de dispensar el depósito también están ventiladas. Menciona la posibilidad de consolar la ejecución provisional en definitiva. Por último, también aborda el cumplimiento provisional de la tutela provisional y los efectos prácticos de la concesión de protección provisional en la sentencia.
\end{abstract}

Abstract

- Keywords:

Provisional execution; Judicial decision; Procedure
Deals with the various aspects related to the provisional execution with judicial decisions in the CPC, focusing on the precedents of the Superior Court of Justice. It addresses the purpose of provisional execution in order to subsequently analyze the hypotheses of suitability

for this type of execution, contrasting them with situations of definitive execution. It deals 
with the rules for processing provisional execution, analyzing the need to provide security, clarifying the exact procedural moment for the practice of that act. The chances of waiving the deposit are also considered. It mentions the possibility of convoluting the provisional execution definitively. Finally, it also addresses the provisional fulfillment of provisional guardianship and the practical effects of granting provisional guardianship in the sentence.

\section{Resumo}

Trata do diversos aspectos relativos ao cumprimento provisório das decisões judiciais no CPC, com enfoque nos precedentes do Superior Tribunal de Justiça. Aborda a finalidade do cumprimento provisório para, em seguida, analisar as hipóteses de cabimento dessa modalidade de execução, contrapondo-as às situações de cumprimento definitivo. Trata das regras de processamento do cumprimento provisório, analisando a necessidade de prestação de caução, esclarecendo sobre o momento procedimental exato para a prática daquele ato. As hipóteses de dispensa da

- Palavras-chave: Cumprimento provisório; Decisão judicial; Procedimento caução também são ventiladas. Menciona a possibilidade de convolação da execução provisória em definitiva. Por fim, aborda ainda o cumprimento provisório da tutela provisória e os efeitos práticos da concessão da tutela provisória na sentença.

1. Introducción - 2. Finalidad de la técnica del cumplimiento provisional de las decisiones judiciales 3. Hipótesis de cumplimiento provisional y definitivo: 3.1. cumplimiento definitivo de una decisión impugnada por "apelação" recibida sin efecto suspensivo; 3.2. cumplimiento definitivo o provisional de la decisión de pronta sentencia parcial del fondo -4 . Procedimiento de la ejecución provisional de la decisión judicial -5 . Provisión de garantía en ejecución provisional -6 . La conversión de la ejecución provisional de decisión judicial en ejecución definitiva -7 . Ejecución de la decisión de tutela provisional y de la tutela provisional concedida en la sentencia -8 . Consideraciones finales Bibliografía

\section{INTRODUCCIÓN}

Con la llegada de la Enmienda Constitucional $n^{\circ} 45$, se insertó en la Constitución Federal el artículo $5^{\circ}$, LXXVIII. Este precepto ha positivado en Brasil el principio de duración razonable de los procedimientos ${ }^{1}$. Esta directriz guió entonces al legislador ordinario, que, por cierto, estableció en el artículo $4^{\circ}$ del nuevo Código de Proceso Civil Brasileño que las partes tienen derecho a obtener en un plazo razonable la solución completa de los méritos, incluida la actividad satisfactoria.

1 Cf. HERTEL, Daniel Roberto. Aspectos processuais da emenda constitucional 45. Revista Forense, Rio de Janeiro, v. 385, mai/jun, 2006. p. 76 e 77. Sobre as tendências do direito processual civil, cf. HERTEL, Daniel Roberto. Perspectivas do direito processual civil brasileiro. Revista dialética de direito processual - RDDP, São Paulo, n. 42, p. 20-30, set., 2006. 
La importancia de la actividad satisfactoria para la eficacia del proceso judicial ha sido reconocida durante mucho tiempo, mencionando que dicha actividad se obtiene precisamente a través del proceso de ejecución o del módulo de cumplimiento de sentencias. De hecho, no tiene sentido reconocer los derechos si esos derechos no se aplican a nivel pragmático. No cabe esperar que el objetivo principal de la jurisdicción, al buscar el Poder Judicial, sea obtener la realización de sus derechos, de modo que cualquier estudio que valore la eficacia de la disposición judicial se armonice con la noción de instrumentalidad del proceso.

En esta oportunidad, se pretende prestar especial atención a las peculiaridades del cumplimiento provisional de las decisiones judiciales esbozadas en los artículos 520 a 522 del Código de Proceso Civil. El cumplimiento provisional de la decisión judicial constituye una técnica procesal capaz de acelerar el instrumento judicial en la realización de los derechos. Su alcance, por lo tanto, es proteger al ciudadano contra demoras procesales indebidas ${ }^{2}$, que a menudo inmortalizan el procesamiento de las demandas.

La demora en el procesamiento de procesos en Brasil es un problema grave y no es raro que un proceso dure, desde la petición inicial hasta la satisfacción del acreedor, muchos años. Por lo tanto, la ejecución provisional (cumplimiento provisional) es una forma de optimizar el desarrollo procesal, con la capacidad de evitar demoras procesales indebidas ${ }^{3}$.

Este artículo toma en cuenta el texto del nuevo Código de Proceso Civil Brasileño (CPC), la doctrina procesal y las decisiones más recientes del Superior Tribunal de Justiça (STJ), responsable por la última palabra sobre la interpretación de la ley federal (no la Constitución) en Brasil. Desafortunadamente, en Brasil no hay datos específicos y cuantitativos sobre la aplicación provisional antes del nuevo Código de Procedimiento Civil para fines de comparación. Sin embargo, esto no constituye un obstáculo para la descripción de un modelo rápido y equitativo de ejecución provisional diante de la nueva ley y ni oportunidad para criticar las eventuales debilidades del nuevo sistema.

2 La doctrina brasileña afirma que "A garantia de duração razoável do processo deve ser compreendida, então, de forma panorâmica, pensando-se na duração total do processo, e não só no tempo necessário para se produzir a sentença do processo de conhecimento" (CÂMARA, Alexandre Freitas. O novo processo civil brasileiro. São Paulo: Editora Atlas, 2015. p. 6).

3 Como destaca la doctrina en Brasil "Pressuposto para aferição da duração razoável do processo é a definição do seu spatium temporis - o dies a quo e o dies ad quem entre os quais o processo se desenvolve. O processo deve ser avaliado para fins de aferição de sua duração, levando-se em consideração todo o tempo em que pendente a judicialização do conflito entre as pares" (MARINONI, Luiz Guilherme. ARENHART, Sérgio Cruz. MITIDIERO, Daniel. Novo código de processo civil comentado. São Paulo: Revista dos tribunais, 2015. p. 97-8). 


\section{FINALIDAD DE LA TÉCNICA DE CUMPLIMIENTO PROVISIONAL DE LAS DECISIONES JUDICIALES}

El cumplimiento provisional de la decisión judicial tiene por objeto acelerar el proceso judicial. De hecho, cuando la Legislatura permitió la ejecución de la decisión incluso antes de su finalización, tenía la intención de acelerar el instrumento judicial. Así, incluso antes de la sentencia definitiva de determinadas decisiones, en las situaciones previstas por la ley, el acreedor puede solicitar la ejecución de las mismas.

Cabe destacar que la técnica procesal de la ejecución provisional está en perfecta armonía con el principio de duración razonable de los procedimientos. Al permitir la ejecución de la decisión antes de su tránsito definitivo, en última instancia, lo que el legislador pretendía era acelerar el procedimiento procesal para satisfacer la ley. Por cierto, el Superior Tribunal de Justicia ya ha declarado que el objetivo de la ejecución provisional es acelerar el procedimiento y antes de la sentencia firme, el acreedor puede solicitarla, en las situaciones previstas por la ley ${ }^{4}$.

\section{HIPÓTESIS DE CUMPLIMIENTO PROVISIONALES Y DEFINITIVAS}

Es muy importante entender en qué casos el cumplimiento de la decisión judicial será provisional y definitivo. El cumplimiento de la decisión judicial será definitivo cuando se base en una decisión judicial dotada de autoridad de cosa juzgada. Cabe señalar que la cosa juzgada se forma cuando la decisión judicial se vuelve inmutable, ya no está sujeta a ningún tipo de recurso ${ }^{5}$. La ejecución basada en un título extrajudicial también será definitiva. En ese orden de ideas, la ejecución de una sentencia que condenó al deudor a pagar una cantidad pecuniaria y que ya ha pasado a ser definitiva (con cosa juzgada ${ }^{6}$ ), será definitiva, al igual que la ejecución de un cheque.

En el otro flanco, el cumplimiento será provisional cuando la decisión judicial sea impugnada por un recurso carente de efecto suspensivo. El efecto suspensivo de la apelación mantiene el estado de ineficacia de la decisión judicial, manteniendo este estado hasta que la apelación sea juzgada definitivamente. Imagínese el cumplimiento

4 STJ, REsp 1686751/DF, Rel. Ministro Moura Ribeiro, Terceira Turma, juzgado en 22-082017, DJe 31-08-2017.

5 La doctrina brasileña explica que "A coisa julgada é instituto cuja função é a de estender ou projetar os efeitos da sentença indefinidamente para o futuro. Com isso, pretende-se zelar pela segurança jurídica extrínseca das relações jurídicas, de certo modo em complementação ao instituto da preclusão, cuja função primordial é garantir a segurança intrínseca do processo, pois que assegura a irreversibilidade das situações jurídicas cristalizadas endoprocessualmente" (WAMBIER, Teresa Arruda Alvim. MEDINA, José Miguel Garcia. O dogma da coisa julgada: hipóteses de relativização. São Paulo: Revista dos tribunais, 2003. p. 21).

6 El professor brasileño Carlos Valder afirma que "A expressão coisa julgada pode ser tomada na acepção de sentença contra a qual foram esgotados todos os recursos processuais passíveis de sua desconstituição" (NASCIMENTO, Carlos Valder (Coord.). Coisa julgada inconstitucional. Rio de Janeiro: América jurídica, 2004. p. 3). 
de una sentencia impugnada por un recurso de casación especial ${ }^{7}$, que, por regla general, se recibe sin efecto suspensivo (artículo 995, CPC) ${ }^{8}$ o incluso de una sentencia condenando el pago de la manutención que ha sido impugnada por "apelação" recibida sin efecto suspensivo (artículo 1012, §1º, inc. II, CPC).

Por cierto, el artículo 520 del Código de Proceso Civil Brasileño establece que la ejecución provisional de la sentencia recurrida mediante recurso de casación carente de efecto suspensivo se llevará a cabo del mismo modo que el cumplimiento definitivo. Así pues, sin el efecto suspensivo del recurso de casación, es posible dar cumplimiento provisionalmente a la resolución judicial.

Por regla general, las apelaciones en los procedimientos civiles son carentes de efecto suspensivo (artículo 995, CPC). De dicha disposición puede extraer que las apelaciones no impiden la eficacia de la decisión, a menos que la disposición legal o la decisión judicial en un sentido diferente.

Sin embargo, parece posible que, debido a una disposición legal o incluso a una determinación judicial, el recurso de casación se reciba con efecto suspensivo. En el primer caso, hablamos del "propio efecto de suspensión", resultante de la disposición legal, mientras que en el segundo, el "efecto suspensivo impropio" se debe a una decisión judicial. En cualquier caso, el efecto suspensivo se atribuirá cuando la eficacia de la Decisión impugnada pueda suspenderse por decisión del ponente, si la producción inmediata de sus efectos existe un riesgo de daño grave, difícil o imposible de reparar, y se demuestra la probabilidad de la presentación del recurso de casación (artículo 995, CPC).

A este respecto, el Superior Tribunal de Justicia (STJ) ya ha declarado que la apelación especial no tiene efecto suspensivo y, por lo tanto, debe aceptarse la ejecución provisional de la sentencia recurrida; por lo tanto, el presente Tribunal ha admitido, en circunstancias excepcionales, la concesión de efectos suspensivos a los recursos de casación de su competencia, mediante tutela provisional, siempre que se

7 La casación especial (recurso especial) se dirige para el Superior Tribunal de Justiça (STJ) y lo que se busca es la preservación de la ley federal. No es un recurso destinado al examen de pruebas o aspectos constitucionales.

8 El artículo 1.029, $\S 5^{\circ}$ del CPC establece que "O pedido de concessão de efeito suspensivo a recurso extraordinário ou a recurso especial poderá ser formulado por requerimento dirigido: I - ao tribunal superior respectivo, no período compreendido entre a publicação da decisão de admissão do recurso e sua distribuição, ficando o relator designado para seu exame prevento para julgá-lo; II - ao relator, se já distribuído o recurso; III - ao presidente ou ao vice-presidente do tribunal recorrido, no período compreendido entre a interposição do recurso e a publicação da decisão de admissão do recurso, assim como no caso de o recurso ter sido sobrestado, nos termos do artículo 1037".

9 La "apelação" se dirige a los tribunales locales y permite la discusión sobre pruebas y la ley. No se confunde con el recurso de casación especial (recurso especial), que se dirige al Superior Tribunal de Justiça y no permite la discusión con respecto a las pruebas. 
cumplan los requisitos de la alta verosimilitud de la reclamación y del peligro de retraso ${ }^{10}$.

Tenga en cuenta que la "apelação" no sigue la regla general sobre la ausencia de efecto suspensivo. El efecto suspensivo sistemático de tal recurso de casación se anula, es decir, en regla general, el recurso de casación tiene efecto suspensivo, estando desprovisto de este efecto únicamente en las hipótesis previstas por la ley.

Por cierto, el artículo 1.012 del CPC establece que la "apelação" tendrá efecto suspensivo, mientras que el primer párrafo dispone que además de otras hipótesis previstas por la ley, comienza a surtir efecto inmediatamente después de su publicación la sentencia que: I - aprueba la división o demarcación de la tierra; II condena a pagar por el mantenimiento; III - extingue sin resolución de los méritos o descarte los embargos de los ejecutados; IV - hasta el mismo tiempo que la solicitud de una institución de arbitraje está bien fundada; V - confirma, concede o revoca la tutela provisional; VI - decreta la prohibición. A continuación, en los casos previstos en el primer párrafo del artículo 1.012 del CPC, ya que el recurso de casación no tiene efecto suspensivo, el demandado podrá solicitar el cumplimiento provisional después de la publicación de la sentencia.

\subsection{Cumplimiento definitivo de una decisión impugnada por "apelação" recibida sin efecto suspensivo}

La cuestión es si una sentencia que ha sido impugnada por "apelação" recibida sin efecto suspensivo está sujeta a su cumplimiento definitivo. De hecho, esto ocurrirá en situaciones excepcionales en las que se llevaría a cabo la sentencia definitiva de una parte de la decisión.

Es cierto que esta cuestión gravita al entorno de la llamada cosa juzgada progresiva, con el tránsito final de capítulos de la decisión judicial que no han sido impugnados, cuya controversia existe en los Tribunales brasileños. El Tribunal Supremo (STF) parece admitir la cosa juzgada progresiva al ver que su resumen 514 establece que la acción de rescisión se acepta contra una sentencia firme, aunque contra ella no se hayan agotado todos los recursos de casación. Por otra parte, el Tribunal Superior de Justicia (STJ) parece no admitir en su mayoría tal posibilidad porque su resumen 401 establece que el término decadente de la acción de rescisión comienza solo cuando no puede apelarse el último pronunciamiento judicial. El artículo 975 del nuevo CPC está alineado con la última orientación.

Imagine una demanda en la que el autor haya reclamado una indemnización por daños morales y materiales. El magistrado desestima las demandas y ordena al demandado que pague tales daños, y el demandado ha presentado una "apelação" especial con el fin de obtener la reforma de la sentencia sólo parcialmente para descartar la condena

10 STJ, AgRg na MC 25.108/SC, Rel. Ministro Napoleão Nunes Maia Filho, Primeira Turma, julgado em 09-06-2020, DJe 23-06-2020. 
de pagar una indemnización por daños morales. Incluso si este recurso de casación ha de ser recibido sin efecto suspensivo, el cumplimiento de la sentencia en relación con el daño material no será provisional, sino definitivo.

Por cierto, el Tribunal Regional Federal de Tercera Región (TRF3) ya ha establecido que la parte no controversial, sobre la que no hay discusión, por lo tanto líquida y segura, está cubierta por el tránsito final, cuidando de la ejecución definitiva, no provisional. En este punto, se operó una resolución parcial de mérito, con formación progresiva de lo juzgado, que permitió el envío de precatorio, en estricta obediencia al artículo 100, párrafo 5, de la Constitución Federal Brasileña ${ }^{11}$. Cabe señalar, sin embargo, que la orientación dominante en el Superior Tribunal de Justicia (STJ) está en la dirección opuesta, es decir, es en el sentido de que debido a que es la acción única e indivisible, no hay necesidad de hablar de fraccionamiento de la frase, lo que elimina la posibilidad de su cosa juzgada parcial, permitiendo su ejecución provisional $^{12}$.

\subsection{Cumplimiento definitivo o provisional de la decisión de juicio anticipado parcial del fondo}

El CPC proporciona en el artículo 356 la posibilidad de juicio parcial del fondo de la demanda, al establecer que el juez decidirá parcialmente el fondo cuando una o más de las solicitudes formuladas o parte de ellas: I - resulte indiscutible; II - está en juicio inmediato, de conformidad con el artículo 355. El segundo y tercer párrafo de dicha disposición establece que la parte podrá resolver o ejecutar, en primer lugar, la obligación reconocida en la decisión de que juzgue parcialmente el fondo, independientemente de la seguridad, incluso si existe un recurso contra esa acción y que En el caso del artículo 2, si hay una sentencia definitiva de la decisión, la ejecución será definitiva.

La disposición incluye la posibilidad de que el Juez tome una decisión parcial sobre el fondo de la demanda. Tradicionalmente, el fondo de la demanda siempre ha tenido su apreciación reservada a la sentencia, que debe dictarse al final del procedimiento. El nuevo Código de Proceso Civil Brasileño, sin embargo, permite el primer análisis meritorio con el juicio anticipado parcial de algunas de las peticiones hechas por el autor.

A este respecto, el Superior Tribunal de Justicia (STJ) ya ha establecido que el nuevo Código de Proceso Civil tiene ahora expresamente la posibilidad de dictar decisiones parciales sobre el fondo, reconociendo la posibilidad de solicitudes acumulativas o porciones de solicitudes que pueden ser fraccionadas, son aptas para el juicio en diferentes momentos procesales, ya sea porque no hay controversia sobre ellos, o

11 TRF $3^{\text {a }}$ Região; AC 0025240-88.2013.4.03.9999; SP; Oitava Turma; Rel ${ }^{\mathrm{a}}$. Des ${ }^{\mathrm{a}}$. Fed. Therezinha Astolphi Cazerta; Julg. 03-11-2014; DEJF 17-11-2014; Pág. 3189.

12 STJ, AgInt no REsp 1489328/RS, Rel. Ministro Benedito Gonçalves, Primeira Turma, julgado em 11-09-2018, DJe 17-09-2018. 
porque no hay necesidad de una mayor dilución probatoria sobre ellas, con aptitud, en ambos casos, para la formación de sentencias con fuerza de cosa juzgada material ${ }^{13}$.

Entonces, ¿será provisional o definitivo el cumplimiento de la decisión de interlocución parcial sobre el fondo? El recurso que se utilizará para impugnar dicho pronunciamiento judicial es el agravo del instrumento. El artículo $356, \S 5^{\circ}$, establece que la decisión adoptada sobre la base del presente artículo se impugna por agravo del instrumento. Por regla general, este recurso no está dotado de efecto suspensivo (artículo 995, CPC). Sin embargo, existe la posibilidad de atribuir su propio efecto suspensivo porque el artículo 1019, I, del CPC, establezco que el relator de la "apelação" podrá atribuir efecto suspensivo a la "apelação" o conceder, en previsión de la tutela, total o parcial, la reclamación recursiva, comunicando al juez su decisión.

Si se concede un efecto suspensivo, no habrá posibilidad de cumplimiento provisional. Por otra parte, si no se concede ningún efecto suspensivo, la decisión entra en vigor y puede estar sujeta a un cumplimiento provisional (ejecución provisional). ¿Qué pasa si no se interpone ninguna "apelação" ante la decisión? Rememoro es un campo con respecto a la cosa juzgada progresista, pero de cualquier arte de la suerte establece el artículo $356, \S 3^{\circ}$ de lo CPC que en el caso del artículo 2 , si hay un tránsito definitivo de la decisión, la ejecución será definitiva.

\section{PROCEDIMIENTO DE LA EJECUCIÓN PROVISIONAL DE LA DECISIÓN JUDICIAL}

El cumplimiento provisional de la decisión judicial se llevará a cabo de conformidad con lo dispuesto en el artículo 520, del Código de Proceso Civil Brasileño. Este preceptivo, en términos generales, dispone que el cumplimiento provisional de la decisión judicial se llevará a cabo de la misma manera que el cumplimiento definitivo, observando las peculiaridades contenidas en los párrafos de dicho artículo.

El artículo 520, I, del CPC establece que el cumplimiento provisional se basa en la iniciativa y la responsabilidad del acreedor, que está obligado, si se reforma la sentencia, a reparar el daño que ha sufrido el ejecutado. Esto significa que el acreedor debe solicitar al Juez el cumplimiento provisional y la iniciación ex officio no es correcta. En este orden de ideas, no es correcto que el Magistrado determine la citación de las partes en relación con el contenido de la sentencia dictada, y la parte es inmediatamente convocada a pagar el importe establecido, bajo pena de multa, en forma de artículo 523, del CPC.

La iniciativa de la parte acreedora también está justificada por el hecho de que la decisión puede ser reformada por el Tribunal de Justicia y cualquier daño causado a

13 STJ, REsp 1798975/SP, Rel. Ministra Nancy Andrighi, Tercera Clase, juzgado el 02-042019, DJe 04-04-2019. Este precedente también conduce a la idea de la formación de cosa juzgada progresista, que está fuera de lugar con la orientación misma del Tribunal Superior de Justicia resultante de la "súmula" 401. 
la ejecución debe ser reembolsado. Por lo tanto, las pérdidas y daños que puedan llevarse a cabo en virtud del cumplimiento provisional deben ser reparados ${ }^{14}$.

Esta responsabilidad es objetiva, sin tener necesidad de la demostración de culpabilidad. Por cierto, el Superior Tribunal de Justicia ya ha declarado que ante la posibilidad de modificar el título judicial que sustenta la ejecución provisional, se impone al acreedor la responsabilidad objetiva de reparar los daños causados al deudor, devolviendo a las partes al estado anterior ${ }^{15}$. Por ejemplo: si el vehículo de un deudor es incautado en caso de cumplimiento provisional, cualquier daño derivado del alquiler de un coche por la parte ejecutada debe ser reparado por el acreedor en caso de reforma de la decisión.

Otra peculiaridad del cumplimiento provisional es que no tendrá ningún efecto mediante una sentencia que modifique o anule la decisión en ejecución (artículo 520, II, CPC). La ejecución es provisional porque hay una "apelação" pendiente. Si se confirma dicha "apelação" del deudor, la ejecución no tendrá efecto. De hecho, cualquier acto procesal perpetrado en el cumplimiento provisional no tendrá efecto, es decir, perderá su eficacia.

Si la ejecución provisional es nula, las partes serán restauradas al estado anterior, es decir, el statu quo ante. En este orden de ideas, cualquier pago que haya sido realizado por el ejecutado en el cumplimiento provisional, debe ser reembolsado por el acreedor. Tenga en cuenta que en el artículo 520, II, del CPC, la Legislatura mencionó la restitución de las "partes" y no la restitución de las "cosas" al Estado anterior. La distinción es relevante porque como sólo las partes serán devueltas al Estado anterior, cualquier disposición del bien que se haya llevado a cabo en el cumplimiento provisional será válida, ya que se reforma la sentencia. Si el Legislador hubiera hecho mención de la devolución de las "cosas" al estado anterior, la alienación tendría que ser deshecha.

También debe señalarse que las pérdidas experimentadas por el ejecutado, sean materiales o morales, se liquidarán en el mismo proceso. No hay necesidad de proponer nueva demanda. La parte perjudicada debe solicitar, en el mismo proceso, por simple petición, la apuración de los daños sufridos.

Si la sentencia sujeta a la ejecución provisional se modifica o anula sólo en parte, la ejecución en la parte modificada o anulada no tendrá ningún efecto (artículo 520, III, CPC). Por ejemplo: se condena al deudor a pagar $\mathrm{R} \$ 5.000,00$ como daño moral y $\mathrm{R} \$$ 5.000,00 como daño material. El deudor, entonces, insatisfecho con la decisión, presenta una "apelação" que se recibe sin efecto suspensivo. El acreedor requiere el cumplimiento provisional (ejecución). El Tribunal confirmó el recurso de "apelação"

14 Dinamarco señala que "en la disciplina de la ejecución provisional, la idea de los procedimientos civiles se manifiesta claramente como un sistema de certezas, probabilidades y riesgos" (DINAMARCO, Cándido Rangel. Reforma de la reforma. 2. ed. Sao Paulo: Malheiros, 2002. página 255).

15 STJ, REsp 1576994/SP, Rel. Ministro Marco Aurélio Bellizze, Tercera Clase, juzgado en 21-11-2017, DJe 29-11-2017. 
para desestimar la parte de la condena relativa al daño moral. En este caso, la ejecución quedará sin efecto sólo en parte, es decir, sólo en relación con la porción de $\mathrm{R} \$ 5000.00$, relativa al daño material.

\section{PROVISIÓN DE GARANTÍA EN EJECUCIÓN PROVISIONAL}

Una de las grandes distinciones entre el cumplimiento provisional y definitivo es que en el primero es necesario proporcionar garantía. Con efecto, dado que se trata de una ejecución provisional y de que hay un recurso pendiente en el Tribunal, es necesario que el acreedor ofrezca una garantía para garantizar cualquier daño que pueda causar a lo ejecutado si se produce una reforma o anulación de la decisión. La garantía, por lo tanto, tiene por objeto garantizar la reparación de las pérdidas que el ejecutado pueda sufrir derivadas del cumplimiento provisional cuándo ocurrir reforma en "apelação".

Cabe señalar, en primer lugar, que no se debe proporcionar la garantía cuando se presenta la solicitud de ejecución provisional. De hecho, la garantía debe proporcionarse en situaciones específicas del procedimiento de cumplimiento provisional, estableciendo el artículo 520, IV, CPC, que la retirada de un depósito en efectivo y la práctica de actos que importen la transferencia de propiedad o enajenación de bienes $u$ otros derechos reales, o de los que puedan derivarse daños graves a los ejecutados, dependen de una garantía suficiente y adecuada, arbitrada por el juez y proporcionada en el propio expediente. Existe la posibilidad de que el procedimiento de ejecución provisional prosiga sin la provisión de una garantía hasta la práctica de actos efectivamente satisfactorios.

Queda claro que no es necesario que la parte ejecutada propone pedido de garantía. Por lo tanto, el magistrado puede determinar de oficio que el acreedor proporciona la seguridad en cumplimiento provisional, conclusión, además, que puede extraerse del arte. 520, IV, CPC, que establece que la garantía será "arbitrada por el juez".

La prestación de la garantía en la ejecución provisional es una regla general. El artículo 521, CPC, sin embargo, non exige garantía en los siguientes casos: el crédito es de naturaleza alimentaria, independientemente de su origen; II - el acreedor demuestra una situación de necesidad; III - el agravo del art. 1.042; y IV - la sentencia que ha de ejecutarse provisionalmente está en consonancia con el resumen de la jurisprudencia del Supremo Tribunal Federal o del Tribunal Superior de Justicia o de conformidad con la sentencia dictada en la sentencia de casos repetitivos.

Hay otra hipótesis en que non se exige garantía en el cumplimiento provisional de la decisión judicial: en el caso de la pronta sentencia parcial del fondo, la provisión de seguridad (art. 356, $\S 2^{\circ}, \mathrm{CPC}$ ). Sin embargo, el requisito de garantía se mantendrá, en cualquier caso, cuando la ejecución pueda dar lugar a un riesgo manifiesto de daños graves de reparación difícil o incierta (artículo 521, párrafo único, CPC). 


\section{LA CONVERSIÓN DE LA EJECUCIÓN PROVISIONAL DE DECISIÓN JUDICIAL EN EJECUCIÓN DEFINITIVA}

El cumplimiento provisional debe solicitarse mediante una petición dirigida al tribunal competente y corresponde al acreedor, al no ser en el proceso electrónico, recoger copias de los siguientes documentos: I - decisión; II - certificado de presentación de la "apelação" no dotada de efecto suspensivo; III - poder otorgado por las partes; IV - decisión habilitante, si procede; y $\mathrm{V}$ - opcionalmente, otros documentos de procedimiento que se consideren necesarios para demostrar la existencia de la reclamación. Dichas copias deben ser autenticadas, pero el propio abogado puede autenticarlas.

El cumplimiento provisional puede convertirse en definitivo. Se dice que el cumplimiento es provisional cuando está respaldado por una decisión que ha sido impugnada por una "apelação" que no tiene efecto suspensivo. Si se juzga la "apelação" y no se interpone ninguna nueva "apelação" dentro del plazo legal, la decisión se convertirá en definitiva, formando res judicata. Por lo tanto, el cumplimiento de la decisión judicial, que puede iniciarse como provisional, debe convertirse en definitivo. En la práctica, el juez debe poner en libertad la garantía que se proporcionó, ya que el régimen de ejecución se convirtió en otro - el de lo definitivo.

\section{EJECUCIÓN DE LA DECISIÓN DE TUTELA PROVISIONAL Y DE LA TUTELA PROVISIONAL CONCEDIDA EN LA SENTENCIA}

La tutela provisional es un género y son especies de esa la de urgencia y la de evidencia. La primera está vinculada a la existencia de periculum in mora y la segunda, no. También vale la pena mencionar que la protección provisional de urgencia puede concederse en carácter antecedente o incidente (artículo 294, CPC). En ese caso (antecedente), la tutela provisional se solicita incluso antes del establecimiento del módulo procesal para la concesión de la tutela principal, mientras que en este último (incidente) se solicita en la propia petición inicial o mediante simple petición durante el procedimiento, sin módulo procesal previo.

Establece el artículo 297, párrafo único, CPC que la eficacia de la tutela provisional se ajustará a las normas relativas al cumplimiento provisional de la sentencia. Por lo tanto, si se concede protección provisional, ya sea de urgencia o de evidencia, su ejecución seguirá las normas sobre el cumplimiento provisional.

A este respecto, el Superior Tribunal de Justicia ya ha aclarado que el Código de Proceso Civil Brasileño de 2015 adoptó la teoría del riesgo-beneficio, estableciendo que el beneficiario con la concesión de la protección provisional debe soportar el daño causado a la parte desfavorable, siempre que: i) la sentencia sea desfavorable para él; (ii) la parte solicitante no proporciona los medios para la citación del demandado en un plazo de cinco (5) días; iii) la eficacia de la medida se produce en cualquier caso legal; o iv) el juez acepta la decadencia o prescripción de la reclamación del autor 
(CPC/2015, artículo 302, I a IV $)^{16}$. En tales casos, cabe mencionar que la indemnización, siempre que sea posible, se resolverá en el proprio proceso en el que se haya concedido la medida.

En el otro flanco, debe señalarse que si el magistrado desea permitir el cumplimiento provisional de una sentencia que pueda ser impugnada por "apelação" con efecto suspensivo, deberá conceder, si no lo ha hecho antes, una protección provisional en la propia sentencia. Por lo tanto, la interposición de cualquier recurso de "apelação" interpuesto ante dicho pronunciamiento judicial no estará dotada de efecto suspensivo (artículo 1.012, $\S 1^{\circ}, \mathrm{V}, \mathrm{CPC}$ ).

El artículo 1015, I, CPC, contempla el cabimiento del recurso de "agravo de instrumento" contra las decisiones que se refieren a la "tutela provisional", lo cierto es que, a la vista de la sentencia, incluso cuando se concede la tutela provisional, el recurso que debe utilizarse es el de recurso de "apelação". Por cierto, es la orientación del Tribunal Superior de Justicia que el recurso apropiado contra la anticipación de la tutela concedido en la sentencia es el recurso, recibido únicamente en efecto de retorno ${ }^{17}$.

\section{CONSIDERACIONES FINALES}

El Legislador hizo una profunda reformulación de la legislación procesal civil en Brasil. La reforma procesal tenía, entre otros objetivos, dar eficacia a la disposición constitucional relativa al principio de duración razonable de los procedimientos. La técnica de cumplimiento provisional de las decisiones judiciales es una medida que armoniza con este objetivo.

Sin embargo, al tratar el cumplimiento provisional de las decisiones judiciales, el Legislador, en mi opinión, han sido mucho tímido. Me parece que, en lugar de mejorar la estructura de elaboración de este modo de aplicación, debería haber ampliado los casos en los que se acepta el cumplimiento provisional. De hecho, ha perdido una excelente oportunidad para establecer, por ejemplo, por regla general, la recepción de la "apelação" sin efecto suspensivo, revirtiendo la norma prevista en el artículo 1.012 CPC.

Como dijo, desafortunadamente, no hay datos específicos y cuantitativos sobre la aplicación provisional antes del nuevo Código de Procedimiento Civil Brasileño para fines de comparación. Pero, ciertamente, permitir que un mayor número de situaciones estén sujetas a la ejecución provisional, con la retirada del efecto suspensivo de la apelación, configuraría una forma de cumplir con el principio de duración razonable del procedimiento.

16 STJ, REsp 1770124/SP, Rel. Ministro Marco Aurélio Bellizze, Tercera Clase, juzgado en 21-05-2019, DJe 24-05-2019.

17 STJ, REsp 267.540/SP, Rel. Ministro Humberto Gomes de Barros, Terceira Turma, juzgado en 21-11-2006, DJ 12-03-2007, p. 217. 


\section{BIBLIOGRAFÍA}

ALVIM WAMBIER, Teresa Arruda et al.: Breves comentários ao novo Código de Processo Civil. São Paulo: Revista dos Tribunais, 2015.

ASSIS, Araken: Cumprimento da sentença. Rio de Janeiro: Forense, 2006.

CÂMARA, Alexandre Freitas: O novo processo civil brasileiro. São Paulo: Editora Atlas, 2015.

CAPPELlETTI, Mauro; GARTH, Bryant: Acesso à justiça. Tradução de Ellen Gracie Northfleet. Porto Alegre: Sérgio Antonio Fabris Editor, 1998.

CARNELUTTI, Francesco: Instituições de processo civil. Tradução de Adrián Sotero de Witt Batista. Campinas: Servanda, 1999. v. 1.

CHIOVENDA, Giuseppe: Instituições de direito processual civil. 3. ed. Tradução de Paolo Capitanio. Campinas: Bookseller, 2002. v. 1.

DINAMARCO, Cândido Rangel: A reforma da reforma. 2. ed. São Paulo: Malheiros, 2002.

DINAMARCO, Cândido Rangel: Instituições de direito processual civil. São Paulo: Malheiros, 2004. v. 4.

DONIZETTI, Elpídio: Curso didático de direito processual civil. 21. ed. rev. atual e ampl. São Paulo: Gen/Atlas, 2018.

HERTEL, Daniel Roberto: Aspectos processuais da emenda constitucional 45. Revista Forense, Rio de Janeiro, v. 385, mai/jun, 2006.

HERTEL, Daniel Roberto: Perspectivas do direito processual civil brasileiro. Revista Dialética de Direito Processual - RDDP, São Paulo, n. 42, p. 20-30, set., 2006.

HERTEL, Daniel Roberto: O processo civil moderno e a dignidade da pessoa humana. Revista Dialética de Direito Processual - RDDP, São Paulo, nº 55, out., 2007.

HERTEL, Daniel Roberto: Curso de execução civil. Rio de Janeiro: Lumen juris, 2008.

LUCON, Paulo Henrique dos Santos: Eficácia das decisões e execução provisória. São Paulo: Revista dos Tribunais, 2000.

MARINONI, Luiz Guilherme; ARENHART, Sérgio Cruz: Execução. São Paulo: Revista dos Tribunais, 2007.

MARINONI, Luiz Guilherme. ARENHART, Sérgio Cruz. MITIDIERO, Daniel: Novo código de processo civil comentado. São Paulo: Revista dos Tribunais, 2015.

MEDIDA, José Miguel Garcia: Novo código de processo civil comentado. São Paulo: Revista dos Tribunais, 2015.

MOREIRA, José Carlos Barbosa: O novo processo civil brasileiro. 23. ed. rev. e atual. Rio de Janeiro: Forense, 2005.

NASCIMENTO, Carlos Valder (Coord.): Coisa julgada inconstitucional. Rio de Janeiro: América jurídica, 2004.

NEVES, Daniel Amorim Assumpção: Manual de direito processual civil. 8. ed. rev. e atual. Salvador: Juspodivm, 2016.

OTEIZA, Eduardo: Prueba pericial y acceso a la justicia. Imparcialidad e igualdad. In: JUNOY, Joan Picó i. VÁZQUEZ, Carlos de Miranda (Coord.). Peritaje y prueba pericial. Barcelona: Bosh editor, 2017. 
THEODORO JÚNIOR, Humberto: Processo de execução. 22. ed. rev. e atual. São Paulo: LEUD, 2004.

THEODORO JÚNIOR, Humberto: Curso de direito processual civil. 41. ed. atual. Rio de Janeiro: Forense, 2007. v. 2.

WAMBIER, Teresa Arruda Alvim; MEDINA, José Miguel Garcia: O dogma da coisa julgada: hipóteses de relativização. São Paulo: Revista dos Tribunais, 2003. 